\title{
229 Development of the trachea and bronchi
}

A The laryngotracheal groove appears at about 25 weeks of embryonic life.

B Approximately 20 million alveoli are present at birth.

C The lung bud forms 3 lobules on the left side.

D The non-respiratory bronchiolar division is complete by birth.

E The number of alveoli does not increase after birth.

\section{In the trachea}

A The carina has an external landmark at the level of the sternal angle.

B A 'forceps space' occurs around a foreign body.

C The mucosal lining is transitional ciliated columnar epithelium.

D The first and second tracheal rings are not infrequently fused.

E The recurrent laryngeal nerve supplies the trachea.

\section{In the neck}

A The recurrent nerves lie in the groove between trachea and vertebral bodies.

B The thymus lies behind the trachea.

C The thyroid isthmus is at a higher level in children than adults.

D Lymphatic drainage is to the deep cervical nodes.

$\mathrm{E}$ The recurrent nerves always pass in front of the inferior thyroid artery.

\section{In the tracheobronchial tree}

A A bronchopulmonary segment consists of a segment of lung with its segmental bronchus.

B Aspiration in a supine patient is most likely to cause problems in the apical bronchus of the right lower lobe.

C The left brachiocephalic vein is anterior to the thoracic trachea.

D Respiratory bronchioles are supported by complete rings of cartilage.

E The carina is $25 \mathrm{~cm}$ from the incisor teeth in an adult. 